\title{
Tenofovir plus entecavir combination therapy for chronic hepatitis $B$ with nucleos(t)ide analogue failure
}

\author{
Bengü Tatar®, Şükran Köse®
}

Department of Infectious Diseases and Clinical Microbiology, University of Health Sciences, İzmir Tepecik Training and Research Hospital, Izmir, Turkey

\begin{abstract}
Objectives: Currently, both entecavir (ETV) and tenofovir (TDF) are recommended first-line therapy for chronic hepatitis B treatment due to good tolerance, lower side-effect profile and high genetic barrier. However, mutations that may develop in the polymerase gene during treatment may result treatment failure. In this study, we aimed to evaluate the efficacy and virologic response of ETV plus TDF combination therapy in chronic hepatitis B patients with suboptimal response to nucleos(t)ide analogues (NAs) monotherapy.

Methods: A total of 813 patients who were followed-up with the diagnosis of chronic hepatitis B and who were treated with TDF or ETV monotherapy were screened. Patients who had a partial or non response to monotherapy during at least 12 months and who was the presence of serum HBV-DNA levels $\geq 2000 \mathrm{IU} / \mathrm{mL}$ at the time of initiation of the ETV plus TDF combination therapy were included.

Results: Ten (1.2\%) patients (9 TDF, 1 ETV) were identified to have had partial response $(50 \%)$ or breaktrough $(40 \%)$ or virologic rebound $(10 \%)$ to monotherapy. The median age was 36.8 years (range, 22-55 years), and $5 / 10(50 \%)$ patients were male. Of 10 patients, nine of achieved undetectable HBV-DNA $(<15 \mathrm{IU} / \mathrm{mL})$ levels ( $50 \%$ of in 6 months and $90 \%$ of in 18 months) with combination therapy. One patient showed no response.

Conclusions: Our results suggest that combination therapy is superior to the antiviral change in treatment failure with NAs. In addition, it is important to conduct HBV drug resistance analyzes to prevent false drug change in treatment.
\end{abstract}

Keywords: Combination therapy, chronic hepatitis B, virologic response, entecavir, tenofovir

$\mathrm{T}$ The main outcome of the chronic hepatitis B therapy is to achieve hepatitis B surface antigen (HBsAg) seronconversion, however this rate is about $1-2 \%$. The second goal of treatment is to prevent progression to cirrhosis and hepatocellular carcinoma, improve quality of life and survival by providing complete virologic response [1]. It is known that high HBV-DNA level is risk factor for disease progression in patients with chronic hepatitis B $[1,2]$. Thus, successful management of patients is important for sup- pressing continuous virologic replication (CVR) and for the remission of liver disease. Nucleos(t)ide analogs (NAs) are the main anti-HBV agents that inhibit HBV replication by targeting the reverse transcriptase region of the hepatitis B virus (HBV) polymerase [3]. However, a long term of treatment may increase the development of drug resistance that can lead to rebound in HBV replication and exacerbation of HBV-related disease [3].

Currently, six NAs are licensed in our country;

How to cite this article: Tatar B, Köse Ş. Tenofovir plus entecavir combination therapy for chronic hepatitis B with nucleos(t)ide analogue failure. Eur Res J 2020;6(4):270-275. DOI: 10.18621/eurj.645852

Address for correspondence: Bengü Tatar, MD., University of Health Sciences, İmir Tepecik Training and Research Hospital, Department of Infectious Diseases and Clinical Microbiology, İmir, Turkey.E-mail: b.gtatar@hotmail.com, Tel: +902324696969 
lamivudine (LAM), adefovir dipivoxil (ADV), entecavir (ETV), telbivudine (LdT), tenofovir disoproxil fumarate (TDF) and tenofovir alafenamide fumarate (TAF). Lamivudine (LAM) was the first oral antiviral agent for chronic hepatitis B. However, the use of LAM is limited because it is associated with a high rate of antiviral resistance and increases up to $65-70 \%$ after 5 years of treatment [4]. For patients with LAM resistant, $\mathrm{ADV}$ has been used as a rescue therapy but, its antiviral effect is not optimal [5]. Among them, both entecavir and tenofovir are recommended firstline therapy for chronic hepatitis B due to good tolerance, lower side-effect profile and high genetic barrier $[6,7]$. The prevalence of ETV resistance in the fiveyear follow-up is only $1.2 \%$. However, it increases to $50 \%$ in patients with lamivudine resistance [8]. TDF resistance was not detected in patients with chronic hepatitis B for 8 years compared with ETV [9].

In this study, we aimed to evaluate the efficacy and virologic response of ETV plus TDF combination therapy in chronic hepatitis B patients with suboptimal response to NAs monotherapy.

\section{METHODS}

\section{Patient Selection}

Between November 2007 - September 2019, a total of 813 patients who were followed-up with the diagnosis of chronic hepatitis B and who were treated with TDF or ETV were screened. The patients who had a partial or non response to TDF or ETV monotherapy during at least 12 months and who was the presence of serum HBV-DNA levels $\geq 2000$ $\mathrm{IU} / \mathrm{mL}$ at the time of initiation of the combination therapy were included in the study. Patients with nonalcoholic fatty liver disease, autoimmune hepatitis, chronic hepatitis $\mathrm{C}$, hepatitis $\mathrm{D}$ virus superinfection, or HIV co-infection were excluded. Patients were received TDF $245 \mathrm{mg}$ and ETV $0.5 \mathrm{mg} / 1 \mathrm{mg}$ once daily.

\section{Laboratory Assessments}

$\mathrm{HBsAg}, \mathrm{HBeAg}$, anti $\mathrm{HBe}$, anti $\mathrm{HBcIgG}$, Anti $\mathrm{HBs}$, Anti HCV and Anti HDV were studied with ELISA (Liaison, Diasorin, Italy). HBV-DNA levels were studied with real-time polymerase chain reaction (PCR). (COBAS Ampli Prep/COBAS, TaqMan; lower limit of quantification, $15 \mathrm{UL} / \mathrm{mL}$ ). HBV genotypic resistance mutations were assessed by Multiplex PCRRevers Hibridizasyon (Inno-Lipa HBV DR v2. Innogenetics, Belgium) Alanine aminotransferase (ALT), aspartate aminotransferase (AST), total bilirubin (Tbil), direct bilirubin (Dbil) were determined with AU5800 auto-analyzer (Beckman Coulter Inc., CA, USA). Alpha Fetoprotein (AFP) was analyzed with the DxI 800 auto-analyzer (Beckman Coulter Inc., CA, USA). Prothrombin time (PT) was studided by CS-2500 automated coagulation analyzer (Sysmex Corporation, Kobe, Japan). International normalized ratio (INR) was calculated using INR = patient $\mathrm{PT} / \mathrm{mean}$ normal PT formula. The platelet (PLT) was analyzed by Beckman Coulter LH 780 (Beckman Coulter Ireland Inc., Mervue, Galway, Ireland). ISHAK scoring system was used to determine the liver inflammation and fibrosis stages. The serum HBV-DNA level was assessed every three months during the first 12 months.

\section{Definitions}

Virological response is defined as undetectable HBV-DNA with a limit of detection of $15 \mathrm{IU} / \mathrm{mL}$. Primary nonresponse is defined as a decrease in serum HBV-DNA $<1 \log 10$ after three months of therapy. Partial virological response is defined as a decrease in HBV-DNA of $>2 \log 10 \mathrm{IU} / \mathrm{mL}$ but detectable HBVDNA after at least 12 months of therapy. Virological breakthrough is defined as an increase in HBV-DNA of $>1 \log 10 \mathrm{IU} / \mathrm{mL}$ in comparison with the baseline at any time during treatment.

\section{RESULTS}

Of the 813 patients $684(84.1 \%)$ were treatmentnaive, $129(15.9 \%)$ were treatment experienced (42 lamivudine, 22 adefovir and 65 peginterferon) before initiating ETV or TDF monotherapy. Among them, 395 were treated with TDF and 418 were treated with ETV, and virologic response rate was $97.7 \%$ in the TDF group and $99.7 \%$ in the ETV group.

Ten $(1.2 \%)$ patients (9 TDF, 1 ETV) were identified to have had partial response $(50 \%)$ or breaktrough $(40 \%)$ or virologic rebound $(10 \%)$ to monotherapy and were treated with a combination of ETV and TDF. One patient had liver cirrhosis and 9 
Table 1. Baseline characteristics and laboratory values of study patients

\begin{tabular}{lcccccccccc}
\hline Patients & MA & ŞŞ & EP & FG & ŞA & ÖG & EG & KL & CÇ & HA \\
\hline Age (years) & 26 & 42 & 30 & 49 & 50 & 22 & 24 & 55 & 48 & 22 \\
Gender & F & M & F & F & M & M & M & F & F & M \\
\hline Baseline & 3.5 & 9.7 & 1.2 & 1.7 & 1.5 & 5.5 & 7.1 & 2.1 & 9.8 & 6.5 \\
HBV-DNA (IU/mL) & $\times 10^{4}$ & $\times 10^{2}$ & $\times 10^{3}$ & $\times 10^{8}$ & $\times 10^{5}$ & $\times 10^{3}$ & $\times 10^{5}$ & $\times 10^{5}$ & $\times 10^{5}$ & $\times 10^{3}$ \\
HBeAg (S/CO) & + & - & + & + & + & + & + & - & + & + \\
\hline Anti HBe (S/CO) & - & + & - & - & + & - & - & + & - & - \\
ALT (U/L) & 58 & 255 & 71 & 425 & 39 & 32 & 255 & 68 & 57 & 36 \\
\hline AST (U/L) & 56 & 142 & 44 & 233 & 30 & 27 & 95 & 40 & 38 & 29 \\
Bilirubin (mg/dL) & 0.5 & 1.1 & 0.7 & 0.9 & 0.7 & 0.8 & 1.5 & 0.9 & 0.7 & 1.0 \\
\hline Albumin (g/dL) & 4.1 & 5 & 4.4 & 3.3 & 4 & 4.5 & 4.9 & 4.0 & 4.1 & 4.3 \\
PLT (K/uL) & 234 & 239 & 325 & 275 & 142 & 316 & 214 & 141 & 163 & 274 \\
\hline INR & 0.9 & 0.9 & 1.1 & 0.9 & 1 & 1.2 & 1.1 & 0.9 & 0.9 & 1.1 \\
\hline AFP(IU/mL) & 1.6 & 1,8 & 5.4 & 2.9 & 4.1 & 2.5 & 2.9 & 2.4 & 5.3 & 4.8 \\
\hline HAI (Ishak scale) & 9 & 7 & 4 & 8 & 7 & 5 & 7 & 4 & 8 & 3 \\
\hline Fibrosis (Ishak scale) & 2 & 3 & 2 & 3 & 5 & 1 & 3 & 2 & 3 & 2 \\
\hline
\end{tabular}

$\mathrm{F}=$ female, $\mathrm{M}=$ male, $(+)=$ positive, $(-)=$ negative, $\mathrm{HBV}=$ hepatitis $\mathrm{B}$ virus, DNA = deoxyribonucleic acid, $\mathrm{AST}=$ Aspartat aminotransferaz, ALT $=$ alanine aminotransferase PLT $=$ platelet, $\mathrm{INR}=$ international normalized ratio, $\mathrm{AFP}=$ Alpha Fetoprotein, $\mathrm{HAI}=$ Histology Activity Index

patients were non-cirrhotic. The patient with cirrhosis received entecavir $1 \mathrm{~g} /$ day plus tenofovir $245 \mathrm{mg} /$ day. The mean duration of combination therapy was 46.1 months (range, 14-60 months). Baseline characteristics and laboratory values of patients are summarized in Table 1 . The median age was 36.8 years (range, 22-55 years), and 5/10 (50\%) patients were male. The median baseline HBV-DNA was $1.72 \mathrm{E}+6 \mathrm{IU} / \mathrm{mL}(9.7 \mathrm{E}+2-1.7 \mathrm{E}+8)$ and $8 / 10$ patients were $\mathrm{HBe} A g$ positive.

HBV resistance mutation to LAM (rt 80, rt 173, rt 180, rt 204 (lipa v2)) was detected in two patients. No

Table 2. HBV-resistance mutation profiles of patients

\begin{tabular}{lll}
\hline & $\begin{array}{c}\text { Resistance Mutation to LAM } \\
\text { rtL80, rtV173, rtL180, rtM204 } \\
\text { (lipa v2) }\end{array}$ & $\begin{array}{c}\text { Resistance Mutation to ADV } \\
\text { rtA181T/V, rtN236T } \\
\text { (lipa v2) }\end{array}$ \\
\hline Patient 1 (MA) & No resistance mutation & No resistance mutation \\
\hline Patient 2 (ŞŞ) & No resistance mutation & No resistance mutation \\
\hline Patient 3 (EP) & Rt180m, 204v mutation & No resistance mutation \\
\hline Patient 4 (FG) & No resistance mutation & No resistance mutation \\
\hline Patient 5 (ŞA) & No resistance mutation & No resistance mutation \\
\hline Patient 6 (ÖG) & No resistance mutation & No resistance mutation \\
\hline Patient 7 (EG) & No resistance mutation & No resistance mutation \\
\hline Patient 8 (KL) & Rt180m, 204v mutation & No resistance mutation \\
\hline Patient 9 (CÇ) & No resistance mutation & No resistance mutation \\
\hline Patient 10 (HA) & No resistance mutation & No resistance mutation \\
\hline
\end{tabular}

$\mathrm{LAM}=$ lamivudine, $\mathrm{ADV}=$ adefovir dipivoxil 
resistance mutation to ADV ( $\mathrm{rtA} 181 \mathrm{~T} / \mathrm{V}, \mathrm{rt} \mathrm{N} 236 \mathrm{~T}$ (lipa v2) was detected (Table 2). One of the two patients with lamivudine resistance received $1 \mathrm{~g} /$ day ETV and the other one received $0.5 \mathrm{~g}$ /day ETV due to side effects. HBeAg seroconversion was observed in 2 of 8 patients after combination therapy at 4 and 22 weeks. There was no difference between HBeAgpositive and $\mathrm{HBeAg}$ negative patients in the characteristics of virological response; 9/10 (90\%) patients achieved undetectable HBV-DNA $(<15$ $\mathrm{IU} / \mathrm{mL}$ ) levels ( $50 \%$ of in 6 months and $40 \%$ of in 18 months). Virological response was observed in the 60th month in the patient receiving ETV $0.5 \mathrm{~g} /$ day with lamivudine resistance and in the 52th month in the cirrhotic patient. One patient who had no virological response was receiving TDF 245 plus ETV $1 \mathrm{~g} /$ day due to lamivudine resistance.

Combination therapy was well tolerated, and no clinically significant side effects and treatment discontinuations were observed. No ALT/AST flares ( $>3$ upper limit of normal) and increase in creatinine levels were observed during the follow-up period.

\section{DISCUSSION}

The limitation of treatment with NAs is the development of HBV resistance variants that can lead to treatment failure and exacerbation of HBV related disease, especially using low potent and low genetic barrier NAs. Mutations in the polymerase gene may cause cross resistance to other NAs and may result virologic breakthrough, biochemical breakthrough. Today, both ETV and TDF were recommended as first-line therapy for chronic hepatitis B drugs due to high potency and higher genetic barrier. Virologic response rate was found $97.7 \%$ with TDF and $99.7 \%$ with ETV in the patients we followed, partial response $(50 \%)$ or breaktrough $(40 \%)$ or virologic rebound $(10 \%)$ to monotherapy were identified in 10 patients. In a multicenter study evaluating the long-term efficacy of TDF and ETV in NAs naive HBeAg positive patients, no significant difference was found between the two drugs in terms of virologic response and tolerability during 144 weeks follow-up [10]. Similar results were confirmed in other studies [11, 12].
In the present study we analysed the efficacy of TDF plus ETV combination therapy in patients who were failed to TDF or ETV monotherapy due to incomplete response or genotypic resistance. Of patients $90 \%$ were achieved undetectable HBV-DNA $(<15 \mathrm{IU} / \mathrm{mL})$ levels and combination therapy was found to have more suppressive effect on virologic suppression. Chung et al. [13] compared the the efficacy of maintenance TDF monotherapy with TDF plus ETV combination therapy in 201 multidrugresistant chronic hepatitis $\mathrm{B}$ patients who were previously treated with ETV plus TDF combination therapy and achieved complete virologic suppression. Among them, 153 were treated with ETV plus TDF combination therapy and 48 were treated TDF monotherapy. During follow up virologic breakthrough was developed in five patients; one patient in TDF monotherapy group and four patients in combination therapy group and there were no significant differences between groups. They reported that there was no additional risk of virologic breakthrough with TDF monotherapy after complete virologic suppression [13]. Chen et al. [14] compared the efficacy between TDF (212 patients) and TDF plus ETV (196 patients) combination therapy with a poor response to ETV. They reported that combination therapy was not superior to TDF monotherapy after the consideration of the rate of viral supression at weeks 24 and 48 [14]. This finding was supported by a study performed by Kim et al. [15]. They were explored the persistence of CVR on 76 antiviral resistant chronic hepatitis $\mathrm{B}$ patients showing CVR on TDF plus ETV $(\mathrm{n}=52)$, TDF plus LAM $(\mathrm{n}=14)$, and TDF plus LdT $(n=10)$ combination therapy, who were switched to TDF monotherapy. The median duration of combination therapy was 20.8 months and the median follow-up period was 24.7 months after switching to TDF monotherapy and all patients maintained CVR and switching from combination therapy to TDF monotherapy has been reported to be good in in virologically suppressed chronic hepatitis B [15]. Li et al. [16] compared the efficacy of TDF switch therapy in 50 chronic hepatitis B patients with suboptimal response to ADV-based combination therapy. Among them 17, 14 and 19 patients were previously treated with LAM plus ADV, LdT plus ADV and ETV plus ADV, respectively. A total of 41 
patients were treated with TDF and nine with TDF plus ETV and the virologic response at week 48 and 96 was reported 76.0 and $89.8 \%$, respectively. Among them, three patients (two of treated with TDF and one of with TDF plus ETV) had suboptimal response and six patients (five of received TDF and one of received TDF plus ETV) had virologic breakthrough. They reported that TDF switch therapy is efficient and safe for patients with chronic hepatitis B with a suboptimal response to ADV-based combination therapy [16].

HBV resistance mutation to LMV (rt 80, rt 173, rt 180, rt 204 (lipa v2) was detected in two patients. Entecavir dose is recommended $1 \mathrm{mg}$ /day if the patient is lamivudine experienced or or has decompensated cirrhosis [17]. In our patient with lamivudine resistance, the virological response was seen in the patient receiving entecavir $0.5 \mathrm{~g} /$ day, but no response occurred in the patient receiving entecavir $1 \mathrm{~g} /$ day. Non-response to treatment was not affected by lamivudine resistance, but was considered to be associated with history of renal transplantation and receiving immunosuppressive therapy of this patient.

No resistance mutation to ADV (rtA181T/V, rt N236T ( lipa v2) was detected. in the study published by Park et al. [18], 63 chronic hepatitis B patients with genotypic resistance to LAM who showed a suboptimal response to LAM and ADV combination therapy were evaluated. Among patients, 30 were treated with ETV plus ADV and 33 were treated with ETV plus TDF for 12 months. They found $84.8 \%$ virologic response rate in ETV plus TDF group [18]. This result was supported by a recent study that performed by Li et al. [19]. They were analyzed the efficacy of ETV monotherapy versus ETV-TDF combination therapy in 220 LAM resistant chronic hepatitis B patients; 114 patients were treated with ETV monotherapy and 106 were treated with ETVTDF combination therapy for at least 24 months. They reported that combination group was superior to the ETV group in achieving a virologic response [19]. Combination therapy was well tolerated, and no clinically significant side effects and treatment discontinuations were observed. No ALT/AST flares (>3 upper limit of normal) and increase in creatinine levels were observed during the follow-up period. On the other hand, given the economic benefit and ease of use, monotherapy can be a better choice after archieve viral suppression.

\section{CONCLUSION}

In conclusion, our results showed combination therapy was superior in the failure of treatment with nucleos(t)ide analogues. In addition, HBV drug resistance analyzes are important to prevent wrong drug changes in treatment. However, further largescale and long-term follow-up prospective studies are needed to explain these results.

\section{Conflict of interest}

The authors disclosed no conflict of interest during the preparation or publication of this manuscript.

\section{Financing}

The authors disclosed that they did not receive any grant during conduction or writing of this study.

\section{Informed consent}

Informed consent was obtained from all participants included in the study.

\section{REFERENCES}

1. Chen CJ, Yang HI, Su J, Jen CL, You SL, Lu SN, et al. Risk of hepatocellular carcinoma across a biological gradient of serum hepatitis B virus DNA level. JAMA 2006;295:65-73.

2. Iloeje UH, Yang HI, Su J, Jen CL, You SL, Chen CJ; Risk Evaluation of Viral Load Elevation and Associated Liver Disease/Cancer-In HBV (the REVEAL-HBV) Study Group. Predicting cirrhosis risk based on the level of circulating hepatitis B viral load. Gastroenterology 2006;130:678-86.

3. Lok AS, Zoulim F, Locarnini S, Bartholomeusz A, Ghany MG, Pawlotsky JM, et al. Hepatitis B virus drug resistance working group. Antiviral drug-resistant HBV: standardization of nomenclature and assays and recommendations for management. Hepatology 2007;46:254-65.

4. Tenney DJ, Rose RE, Baldick CJ, Pokornowski KA, Eggers BJ, Fang J, et al. Long-term monitoring shows hepatitis B virus resistance to entecavir in nucleoside-naive patients is rare through 5 years of therapy. Hepatology 2009;49:1503-14.

5. Lok AS, McMahon BJ. Chronic hepatitis B. Hepatology 2007;45:507-39.

6. European Association For The Study Of The Liver. EASL Clinical Practice Guidelines: management of chronic hepatitis B. J Hepatol 2009;50:227-42.

7. Dienstag JL. Benefits and risks of nucleoside analog therapy for hepatitis B. Hepatology 2009;49:112-21.

8. Zoulim F, Durantel D, Deny P. Management and prevention of drug resistance in chronic hepatitis B. Liver Int 2009;29:10815. 
9. Liu Y, Corsa AC, Buti M, Cathcart AL, Flaherty JF, Miller MD, et al. No detectable resistance to tenofovir disoproxil fuma-rate in $\mathrm{HBeAg}+$ and $\mathrm{HBeAg}$ _ patients with chronic hepatitis B after 8 years of treatment. J Viral Hepat 2017;24:68-74.

10. Cai D, Pan C, Yu W, Dang S, Li J, Wu S,et al. Comparison of the long-term efficacy of tenofovir and entecavir in nucleos(t)ide analogue-naïve $\mathrm{HBeAg-positive} \mathrm{patients} \mathrm{with} \mathrm{chronic} \mathrm{hepatitis}$ B. A large, multicentre, randomized controlled trials. Medicine (Baltimore) 2019;98:e13983.

11. Ha NB, Trinh HN, Rosenblatt L, Nghiem D, Nguyen MH. Treatment outcomes with first-line therapies with entecavir and tenofovir in treatment-naive chronic hepatitis B patients in a routine clinical practice. J Clin Gastroenterol 2016;50:169-74.

12. Batirel A, Guclu E, Arslan F, Kocak F, Karabay O, Ozer S, et al. Comparable efficacy of tenofovir versus entecavir and predictors of response in treatment-naïve patients with chronic hepatitis B: a multicenter real-life study. Int $\mathrm{J}$ Infect Dis 2014;28:153-9.

13. Chung SW, Chang Y, Lee HY, Cho EJ, Lee JH, Yu SJ, et al. Is combination antiviral therapy mandatory for maintenance therapy in fully suppressed multidrug-resistant hepatitis B patients? Gastroenterol Res Pract 2018;2018:6948235.

14. Chen J, Zhao SS, Liu XX, Huang ZB, Huang Y. Comparison of the efficacy of tenofovir Versus tenofovir plus entecavir in the treatment of chronic hepatitis B in patients with poor efficacy of entecavir: a systematic review and meta-analysis. Clin Ther 2017;39:1870-80.

15. Kim DY, Lee HW, Song JE, Kim BK, Kim SU, Kim DY, et al. Switching from tenofovir and nucleoside analogue therapy to tenofovir monotherapy in virologically suppressed chronic hepatitis B patients with antiviral resistance. J Med Virol 2018;90:497-502.

16. Li X, Luo J, Zhu C, Wu Y, Li Z, Jie Y, et al. Efficacy of tenofovir disoproxil fumarate switch therapy in chronic hepatitis B patients with suboptimal response to adefovir-based combination therapy. Exp Ther Med 2019;17:1196-205.

17. Terrault NA, Lok ASF, McMahon BJ, Chang K-M, Hwang JP, Jonas MM, et al. Update on Prevention, Diagnosis, and Treatment of Chronic Hepatitis B: AASLD 2018 Hepatitis B Guidance. Hepatology 2018;67:1560-99.

18. Park JG, Park SY. Entecavir plus tenofovir versus entecavir plus adefovir in chronic hepatitis B patients with a suboptimal response to lamivudine and adefovir combination therapy. Clin Mol Hepatol 2015;21:242-8.

19. Li W, Wang L, Liu Y, Li Y, Yu S. Efficacy of entecavir-based rescue therapy in lamivudine-resistant chronic hepatitis B patients in China: a retrospective study. Pharmazie 2018;73:223-47. 